\title{
REVIEW
}

\section{Point-of-care coagulation management in intensive care medicine}

\author{
Patrick Meybohm, Kai Zacharowski*, Christian F Weber \\ This article is one of ten reviews selected from the Annual Update in Intensive Care and Emergency Medicine 2013 and co-published as a series \\ in Critical Care. Other articles in the series can be found online at http://ccforum.com/series/annualupdate2013. Further information about the \\ Annual Update in Intensive Care and Emergency Medicine is available from http://www.springer.com/series/8901.
}

\begin{abstract}
Introduction
Coagulopathy in critically ill patients is common and of multifactorial origin [1]. Coagulopathy-associated risk of bleeding and the use of allogeneic blood products are independent risk factors for morbidity and mortality $[2,3]$. Therefore, prompt and correct identification of the underlying causes of these coagulation abnormalities is required, since each coagulation abnormality necessitates very different therapeutic management strategies. Standard laboratory tests of blood coagulation yield only partial diagnostic information, and important coagulation defects, e.g., reduced clot stability, platelet dysfunction, or hyperfibrinolysis, remain undetected. Therefore, point-of-care (POC) diagnostics are increasingly being used for rapid specific testing of hemostatic function. Algorithm-based hemotherapy, including POC techniques, reliably corrects coagulopathy, but may also have the potential to reduce blood loss, transfusion requirements and risk of transfusion-related adverse events, prevent thromboembolic events, and save costs.

This article reviews the most frequent coagulation abnormalities in critically ill patients. In particular, we will discuss differential diagnoses, benefits and limitations of POC coagulation management and hemotherapy algorithms.
\end{abstract}

\section{Diagnosis of coagulopathy in intensive care medicine}

Coagulopathy in critically ill patients is typically a multifactorial problem involving:

- disturbances in physiological basic conditions for hemostasis $(\mathrm{pH}$, concentration of ionized calcium, temperature and hematocrit)

*Correspondence: kai.zacharowski@kgu.de

Clinic of Anesthesiology, Intensive Care Medicine and Pain Therapy, Goethe-University Hospital Frankfurt, Theodor-Stern-Kai 7, 60590 Frankfurt am Main, Germany
- disturbances of primary hemostasis, e.g., preexisting or perioperatively acquired disturbances of platelet count and function, due to sepsis, disseminated intravascular coagulation (DIC), heparin-induced thrombocytopenia, massive blood loss, or drug-induced thrombocytopenia;

- abnormalities of blood plasma, e.g., preoperative anticoagulation medication as well as isolated or global clotting-factor deficits (impaired synthesis, massive loss, or increased turnover);

- complex coagulopathies, e.g., DIC or hyperfibrinolysis (Figure 1) [1].

In patients with extracorporeal life support (ventricular-assist devices, extracorporeal membrane oxygenation [4]), the risk of coagulopathy is further increased by:

- therapeutic anticoagulation with the use of heparin to limit clotting;

- dilution, activation and consumption of both coagulation factors and platelets.

\section{Limitations of conventional laboratory coagulation analyses}

The conventional laboratory coagulation analyses (Quick's test, activated partial thromboplastin time (aPTT), platelet count, fibrinogen concentration) may be of limited use for the prediction and detection of coagulopathies and for treatment monitoring, in particular in patients with ongoing bleeding [5]. Analysis at a standardized temperature of $37^{\circ} \mathrm{C}$ impedes the detection of coagulopathies induced by hypothermia. The global tests (e.g., aPTT and Quick's test) reflect only the initial formation of thrombin in plasma and are unaffected by any of the corpuscular elements of the blood. The platelet count is purely quantitative and cannot detect preexisting, drug-induced, or perioperatively acquired platelet dysfunction. Moreover, conventional coagulation tests convey no information about clot stability over time, nor do they provide any information regarding (hyper-)fibrinolysis. Thus, it is 


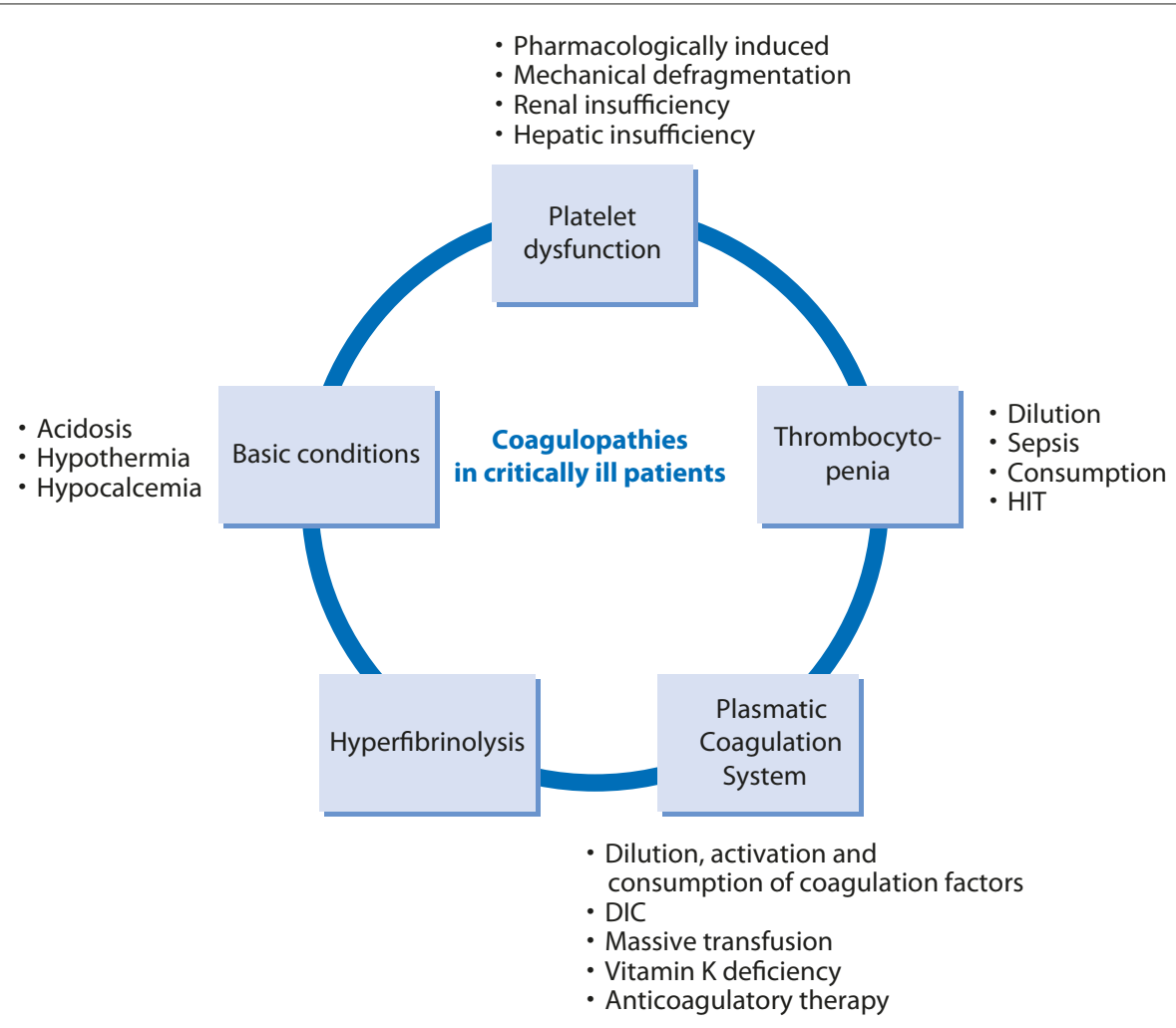

Figure 1. Overview of coagulopathies typically present in critically ill patients. DIC: disseminated intravascular coagulopathy; HIT: heparininduced thrombocytopenia.

critically important to recognize that routine coagulation tests cannot detect clinically significant coagulation defects that contribute to bleeding, hypo- or hyperfibrinolysis, hypercoagulability, and platelet aggregation.

\section{Point-of-care techniques}

In contrast to standard laboratory tests, POC techniques, including whole blood platelet function tests (impedance or turbidimetric aggregometry) and viscoelastic tests (thromboelastometry/-graphy), reflect in detail the hemostatic status of the critically ill patient. The use of POC diagnostics may partly compensate for the methodological limitations of conventional coagulation testing. Test results are also available earlier (analysis time of 20 to 25 minutes [6]) compared to conventional laboratory analyses (turnaround time of 40 to 90 minutes after blood drawing [7]), for which the delayed results may not reflect the current state of the coagulation system and may lead to inappropriate treatment.

However, none of the currently available POC techniques can provide adequate information about all aspects of the complex process of blood clotting. From a pathophysiological point of view, coagulation can be divided into four areas: Primary hemostasis, thrombin generation, clot formation/stabilization, and fibrinolysis.
Aggregometric testing of whole-blood samples is used mainly to study platelet function [8]. In bleeding patients in whom the hematocrit is greater than $30 \%$ and the platelet count exceeds $70-100 / \mathrm{nl}$, aggregometric tests can be used to screen for disorders of primary hemostasis, e.g., von Willebrand syndrome, and to quantify the effect of antiplatelet medications. The available aggregometric POC tests (Multiplate, PFA-100, TEG Platelet Mapping ${ }^{\mathrm{TM}}$ Assay, VerifyNow') differ in the agonists that are used to activate the platelets in the test cells, such as collagen, adenosine phosphate, epinephrine, arachidonic acid, and thrombin, and in the shearing forces that are generated in the test cells.

Viscoelastic POC techniques (ROTEM', TEG) are based on thromboelastography, which was described decades ago by Hartert [9]. These tests are used to measure the time until clot formation begins, the dynamics of clot formation, and the solidity and stability of clots over time. They enable parallel measurements to be performed on a single blood sample after clotting has been activated using a variety of agonists. A special advantage of viscoelastic techniques is that they can directly detect hyperfibrinolysis. Aggregometric tests combined with viscoelastic methods yield a far broader diagnostic spectrum than do conventional laboratory testing of coagulation. 


\section{Implementation of POC testing in intensive care medicine \\ Cardiovascular surgery}

Most of the previous trials related to POC coagulation testing were performed in cardiac surgical patients [1013]. The majority reported a potential decrease in transfusion requirements following POC diagnostics. In two studies, the authors exclusively focused on coagulopathic patients $[13,14]$, in whom POC techniques resulted in significantly reduced postoperative blood loss and beneficial effects in terms of clinically relevant endpoints. Nuttall et al. [14] randomized patients to a control group following individual anesthesiologist's transfusion practices or a protocol group using a transfusion algorithm guided by coagulation tests (prothrombin time, aPTT, platelet counts, thromboelastogram maximum amplitude, and fibrinogen concentration). We recently published the results of a prospective study including coagulopathic patients in whom diffuse bleeding was diagnosed after heparin reversal or increased blood loss was observed during the first 24 hours postoperatively; the previous protocol group of Nuttall et al. was now defined as our control group with conventional tests (platelet count, fibrinogen concentration, international normalized ratio [INR], aPTT, and activated clotting time [ACT]), and patients in the POC-guided group received repeated thromboelastometry and whole blood impedance aggregometry [13]. The following endpoints were lower in the POC-guided group: Erythrocyte, frozen plasma and platelet transfusion rates; postoperative mechanical ventilation duration; length of ICU stay; composite adverse events rate; costs of hemostatic therapy; and 6-month mortality (Figure 2).

Our findings support those of a recently published retrospective study by Gorlinger et al. [15] that included 3,865 cardiac surgery patients in whom POC testing combined with first-line administration of coagulation factor concentrates was associated with decreased incidence of blood transfusion and adverse events. In ICU patients undergoing mechanical circulatory support, monitoring of hemostasis using POC thromboelastometry/-graphy and platelet function analysis is also recommended to reduce the risk of bleeding and thromboembolic complications [4]. Notably, these hemostatic tests should be performed repeatedly during mechanical circulatory support therapy since thrombin generation, clot firmness and platelet response may change significantly over time with marked inter- and intra-individual variability.

\section{Organ transplantation}

In patients with liver transplantation, significant blood loss has been found to be an independent risk factor for postoperative morbidity. To date, there is no consensus as to how to monitor and manage perioperative blood loss. In a prospective randomized study, Wang et al. recently randomized 28 patients undergoing orthotopic liver transplantation either to standard laboratory measures of blood coagulation or thromboelastography analysis. POC-guided transfusion was associated with decreased transfusion of frozen plasma (12.8 \pm 7 vs. $21.5 \pm 12.7$ units), but did not affect 3-year survival [16]. Analyzing more than 18,000 thromboelastography measurements in the context of 642 patients with liver transplantation, the implementation of a POC coagulation management based on early, calculated, goaldirected therapy with fibrinogen concentrate, prothrombin complex concentrate and antifibrinolytic therapy resulted in early detection of hyperfibrinolysis and consequently antifibrinolytic therapy [17], and a reduction in transfusion requirements for erythrocytes, fresh frozen plasma (FFP), and platelets as well as a reduced incidence of massive transfusion [18].

\section{Massive bleeding and trauma patients}

When critically ill patients need urgent massive transfusion because of massive blood loss, there may not be enough time to analyze the results of POC testing before the erythrocyte transfusion is given. However, coagulation factor deficiency is the primary cause of coagulopathy in massive transfusion because of dilution of coagulation factors. The level of fibrinogen typically falls below the lower reference range after $150 \%$ blood volume loss, followed by a decrease in other coagulation factors to $25 \%$ activity after $200 \%$ blood loss [19]. FFP alone, if given in sufficient quantity ( $>15-20 \mathrm{ml} / \mathrm{kg}$ ), will most likely correct fibrinogen and most coagulation factor deficiencies, but large volumes may be required, which are further associated with infectious complications [20] and multiple organ failure [21]; therefore goal-directed therapy with coagulation factor concentrates should be preferred [18] in patients outside the clinical setting of 'massive transfusion'.

In major trauma patients, coagulopathy has been shown to be present in approximately $30 \%$ of admitted patients, accounting for up to $40 \%$ of all trauma-related deaths [22]. Although FFP transfusion is often a routine part of transfusion protocols, its efficacy is uncertain. Most notably, FFP administration is also associated with acute lung injury (ALI), volume overload, and nosocomial infection [20,21]. Additionally, trauma-associated coagulopathy is the consequence of a low admission fibrinogen level, which is independently associated with injury severity score, shock, and mortality at $24 \mathrm{~h}$ and 28 days after trauma. In a prospective cohort study of 517 trauma patients, thromboelastography enabled rapid detection of hypofibrinogenemia, and early administration of fibrinogen was related with improved survival [23]. Using rapid thromboelastography, Holcomb et al. recently succeeded 

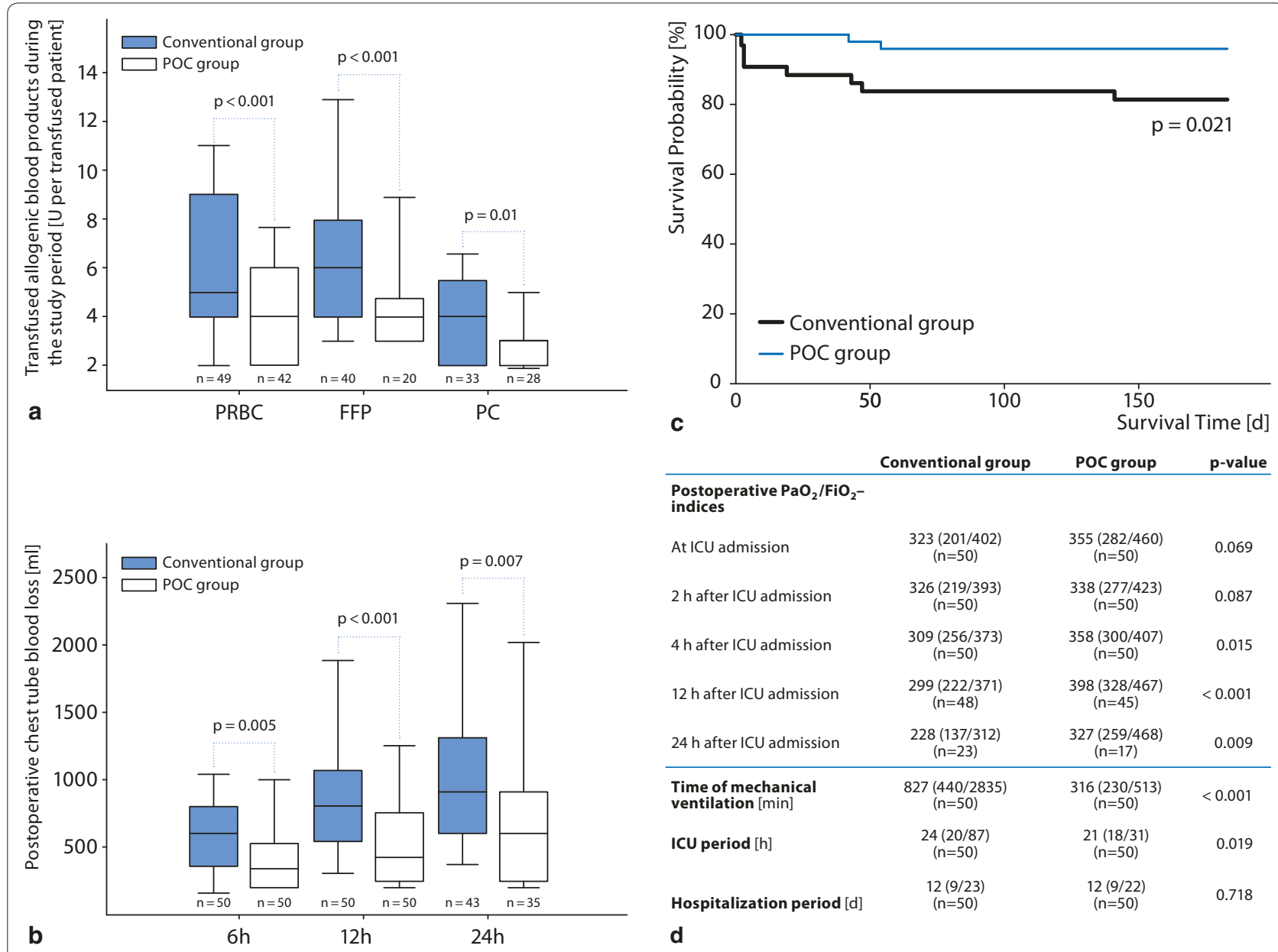

b

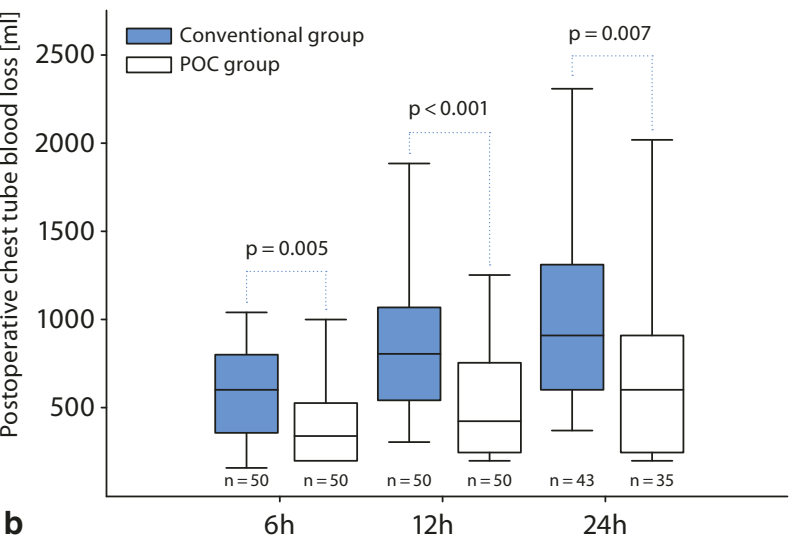

d

Figure 2. Primary and secondary outcomes of the first prospective randomized study in complex cardiac surgery including viscoelastic and aggregometric measures into a point-of-care (POC)-based algorithm for hemotherapy as proof-of-concept [13]. (a) Packed red blood cells (PRBC), fresh frozen plasma (FFP) and platelet concentrate (PC) transfusion rate; (b) postoperative chest tube blood loss during the first $24 \mathrm{~h}$ after admission to the intensive care unit; (c) mortality during a 6-month follow up observation period; and (d) ventilation time, $\mathrm{PaO}_{2} / \mathrm{FiO}_{2}-$ index, and length of stay in the intensive care unit and hospital. From [13] with permission.

in identifying patients with an increased risk of fibrinolysis and erythrocyte, plasma and platelet transfusions out of 1,974 major trauma patients [24]. Schochl et al. [25] retrospectively analyzed the effects of thromboelastometry-guided hemostatic therapy in 131 trauma patients who received $\geq 5$ erythrocyte concentrate units within 24 hours after admission. POC-guided hemostatic therapy with fibrinogen concentrate as first-line hemostatic therapy (if maximum clot firmness [MCF] measured by FibTEM [fibrin-based test] was $<10 \mathrm{~mm}$ ) and additional use of prothrombin complex concentrate (if clotting time measured by extrinsic activation test [EXTEM] $>1.5$ times normal) allowed rapid and reliable diagnosis of the underlying coagulopathy, and resulted in a favorable survival rate compared with predicted mortality [25]. Although a recent meta-analysis concluded that use of a thromboelastography- or thromboelastometry-guided transfusion strategy significantly reduced bleeding in massively transfused patients, these POC techniques were not, however, able to improve morbidity or mortality in patients with massive transfusion [26]. Therefore, prospective randomized studies focusing on POC-guided therapy are needed in these patients.

\section{Severe sepsis}

Because hemostatic alterations are a common early event in patients with severe sepsis, and commonly used sepsis biomarkers, such as procalcitonin and interleukin (IL)-6, may also increase in patients with trauma or surgery even without infection, thromboelastometry variables may have potential as early biomarkers of sepsis in critically ill patients. Adamzik and colleagues [27] recently demonstrated, in an observational cohort study of 56 patients with severe sepsis and 52 patients after major surgery, 
that the thromboelastometry-derived lysis index was a more reliable biomarker of severe sepsis than were procalcitonin, IL-6, and C-reactive protein (CRP). More interestingly, a multivariate analysis of another cohort study of 98 septic patients [28] revealed that the absence or presence of at least one pathological thromboelastometry variable allowed better prediction of 30-day survival in severe sepsis than did the simplified acute physiology system (SAPS) II and sequential organ failure assessment (SOFA) scores, emphasizing the importance of the coagulation system and the future role of POC techniques in sepsis.

\section{Economic aspects}

Results of the currently available trials do not allow any firm conclusions about the putative economic savings resulting from the use of $\mathrm{POC}$ coagulation testing. A number of retrospective studies have compared the costs of hemotherapy before and after the implementation of POC-based hemotherapy algorithms, albeit with partially conflicting results. In a study of 1,422 patients undergoing elective cardiac surgery, Spalding et al. found that implementation of POC coagulation testing lowered the cost of allogeneic blood products and other hemotherapeutic agents by about $50 \%$ [29]. The total cost of POC testing (devices, reagents, test tubes, control solutions, maintenance, etc.) exceeds that of conventional coagulation testing, because combined viscoelastic and aggregometric coagulation testing costs about $€ 25$ to $€ 35$, whereas a conventional test battery (aPTT, fibrinogen, thrombin time, Quick, complete blood count) generally costs less than $€ 10$. Taken together, it appears that POC-guided coagulation therapy indeed lowers the rate of transfusion of allogeneic blood products overall (mainly by lowering FFP and platelet transfusion rates), but simultaneously increases the use of clotting-factor concentrates (mainly fibrinogen and prothrombin complex concentrates) [15]. The economic savings from the reduced use of allogeneic blood products, may, however, compensate for or outweigh these increased expenditures in clotting-factor concentrates [13].

\section{Hemotherapy algorithm including point-of-care techniques}

Coagulation management in intensive care medicine should be based on a hemotherapy algorithm that includes POC techniques, and that is implemented as institutional standard care (Figure 3). Hemotherapy should involve an assessment of the patient's individual bleeding risk, evaluation and correction of basic physiological conditions required for hemostasis, and repeated evaluation and correction of thrombin and clot formation. If indicated, replacing deficient coagulation factors and improving the hemostatic potential of the primary hemostasis are consecutive steps in therapy escalation. Final options are off label use of factor XIII and recombinant factor VIIa.

\section{Maintain optimal physiological conditions for hemostasis}

The patient's physiological $\mathrm{pH}$ and core temperature should be maintained at $>7.3$ and $>36^{\circ} \mathrm{C}$, respectively. Calcium is an elementary cofactor in several enzymatic processes during coagulation. It is important to maintain a plasma ionized calcium concentration of $>1.0 \mathrm{mmol} / \mathrm{l}$. With respect to the rheological properties of erythrocytes and the provision of thromboxane A2 and adenosine diphosphate (ADP) for platelet aggregation, hematocrit should be maintained at a concentration of $>25 \%$ [19].

\section{Antagonize anticoagulant therapy}

In patients with previous heparin therapy (e.g., extracorporeal life support, renal replacement therapy) and ongoing bleeding, it is necessary to exclude any persistent heparin effects. Protamine is routinely used to antagonize heparin. However, if the ACT remains $>130$ s or viscoelastic measures indicate persistent heparin effects, it may be necessary to administer additional protamine $(30 \mathrm{IU} / \mathrm{kg})$ and to re-evaluate its therapeutic effect.

Newer anticoagulants include both direct and indirect inhibitors of coagulation factors. The indirect (antithrombin-dependent) inhibitors include the low molecular weight heparins (e.g., enoxaparin and dalteparin) and selective factor $\mathrm{Xa}$ inhibitors (e.g., fondaparinux and rivaroxaban). The direct thrombin inhibitors (e.g., lepirudin, argatroban, bivalirudin, and dabigatran) directly bind to and inhibit thrombin. Approximately $60 \%$ of the anticoagulant effect of low-molecular-weight heparins can also be neutralized by protamine. In contrast, there are no specific reversal agents for any of the newer anticoagulants; prothrombin complex concentrates might be beneficial for the treatment of anticoagulant-associated coagulation factor deficiencies (dose ranges from 25 to $100 \mathrm{U} / \mathrm{kg}$ depending on the product used). In patients with life-threatening bleeding, hemodialysis might be considered to remove selected small-molecule anticoagulants (e.g., lepirudin, dabigatran) [30].

\section{Maintain adequate levels of coagulation factors}

If coagulopathy persists following reversal of the anticoagulant effects, it should be ensured that adequate levels of coagulation factors are maintained. Fibrinogen is the first coagulation factor to decrease below lower reference values during bleeding [31]. As the precursor to fibrin, and as a ligand for platelet aggregation, fibrinogen plays a key role in clot formation [32]. Thus, if plasma levels of fibrinogen decrease below $150-200 \mathrm{mg} / \mathrm{dl}$, or if viscoelastic tests indicate a deficiency (if maximum clot firmness measured by FibTEM is $<10 \mathrm{~mm}$ ), it is necessary 


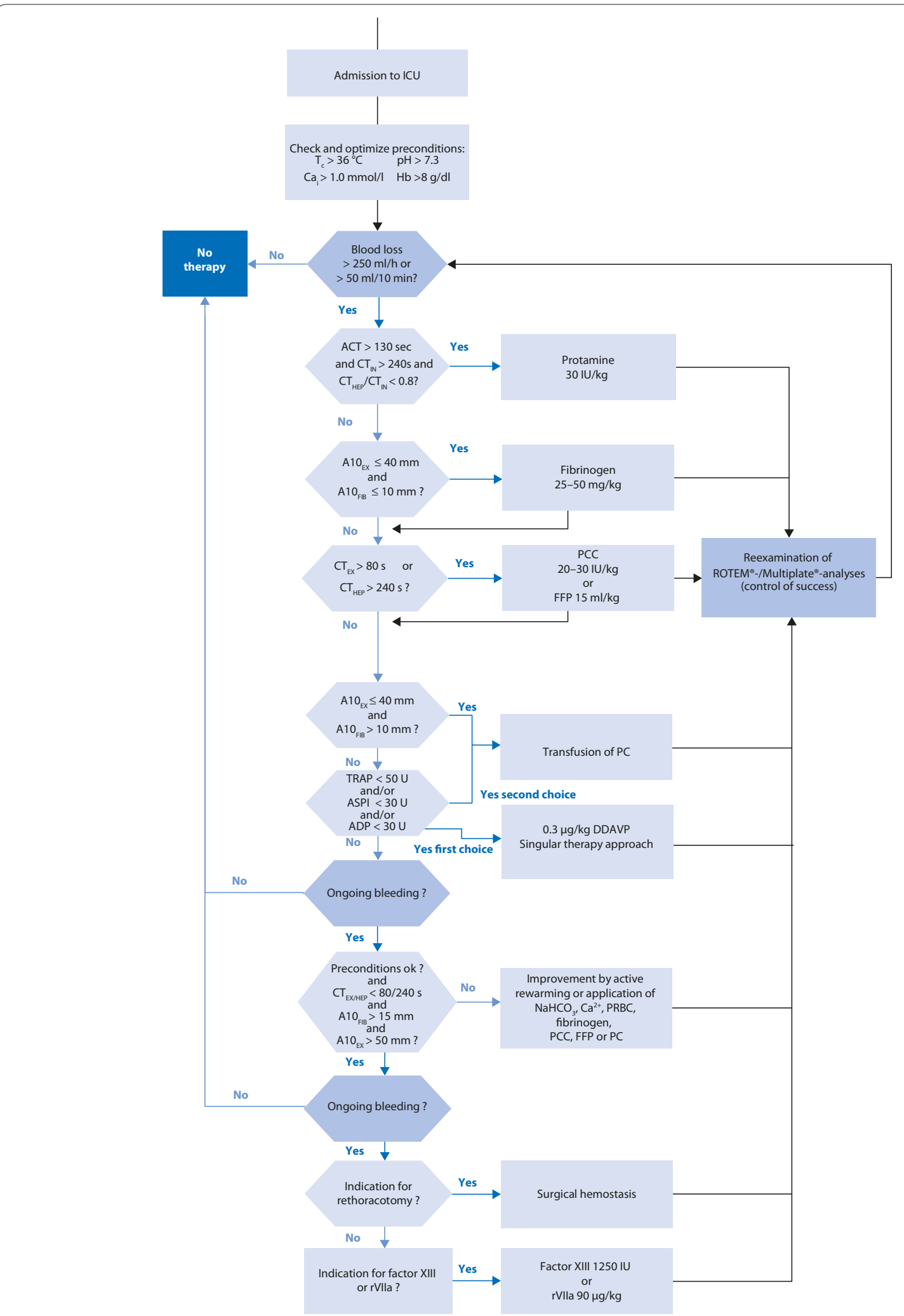

Figure 3. Hemotherapy algorithm including point-of-care (POC) techniques. ACT: activated clotting time; ADP: ADPtest; ASPI: ASPItest; A10: amplitude of clot firmness 10 min after clotting time; Ca: ionized calcium; CT: clotting time; EX: EXTEM; FFP: fresh frozen plasma; FIB: FIBTEM; F XIII: factor XIII concentrate; HEP: HEPTEM; ICU: intensive care unit; IN: INTEM; PC: pooled platelet concentrate; PCC: prothrombin complex concentrate; rFVIla: activated recombinant factor VIl; $T_{c}$ : core temperature; TRAP: TRAPtest. From [13] with permission. 
to administer fibrinogen substitute. In the past, FFP $(15-30 \mathrm{ml} / \mathrm{kg})$ was used as a source of replacement fibrinogen. But again, the transfusion of FFP is, under certain conditions, associated with volume overload, sepsis, multiple organ failure, and increased perioperative mortality [20,21]. An alternative source of fibrinogen is fibrinogen concentrate, which should be used at a concentration of $25-50 \mathrm{mg} / \mathrm{kg}$. A recent review of trials in which fibrinogen concentrate was used in perioperative settings or in cases of massive hemorrhage suggests that this form of substitution is both effective and well tolerated [33].

If coagulopathy persists even though fibrinogen has been supplemented, and if the INR is $>1.4$, or if viscoelastic measures reveal a deficiency of prothrombincomplex coagulation factors II, VII, IX, and X, then substitution of these factors is indicated. A dosage of 15$30 \mathrm{ml} / \mathrm{kg}$ FFP is necessary to increase the concentration of these factors. However, the use of a prothrombincomplex concentrate, which contains factors II, VII, IX, and $\mathrm{X}$, proteins $\mathrm{C}$ and $\mathrm{S}$, heparin, and antithrombin, represents an attractive alternative because of the smaller volumes required to supplement the deficiency (recommended dose 20-30 IU/kg) [34].

\section{Platelets}

After the substitution of coagulation factors, the therapy algorithm should lead to a consideration of both platelet count and function. Importantly, temperature has a strong impact on platelet function [35]. Primary hemostasis may further be impaired by preexisting or perioperatively-acquired disturbances of platelet function, or isolated thrombocytopenia from sepsis, DIC, heparininduced thrombocytopenia, massive blood loss, or druginduced thrombocytopenia. In addition, use of extracorporeal circulation may also cause irreversible platelet damage mainly as a result of mechanical defragmentation and reduced platelet surface glycoproteins (GPIb and GPIIb/IIIa), which are important for platelet adhesion and aggregation [36].

Routine standard laboratory analyses are of limited informative value as they only provide a quantitative measure of platelet numbers without providing any information regarding platelet functionality. In contrast, aggregometric measures allow assessment of platelet function by measuring the amount of platelet aggregation induced in the presence of common platelet agonists such as thrombin, arachidonic acid, ADP, epinephrine, or collagen. Aggregometric tests help to identify surgical patients at increased risk of bleeding or with resistance to anti-platelet therapy and, therefore, at increased risk of thromboembolism. Moreover, these tests may help to analyze the extent of platelet dysfunction in critically ill patients [37]. However, the clinical outcome benefits of including these tests in hemotherapy algorithms have not yet been investigated.

If platelet function has been shown to be deficient, administration of desamino-D-arginine vasopressin (DDAVP) represents a therapeutic option. DDAVP $(0.3 \mu \mathrm{g} / \mathrm{kg})$ has been shown to induce a three-fold increase in von-Willebrand factor and factor VIII [38]. These factors promote an increase in platelet-endothelial cell adhesion via the GPIb receptor and platelet-platelet aggregation via the GPIIb/IIIa receptor. Hemostatic potential can further be improved by transfusion of platelet concentrates; one unit of platelet concentrate increases the platelet count by approximately 30,000/ $\mu \mathrm{l}$. However, there are inherent risks associated with the transfusion of platelet concentrates, in particular risk of bacterial and/or viral infections. Additionally, the number and functionality of platelets in platelet concentrates decreases with storage time [39].

\section{Ultima ratio}

If coagulopathy persists and surgical causes for bleeding have been ruled out, off-label use of two additional coagulation factor concentrates, factor XIII and activated factor VII, may represent an 'ultima ratio' therapeutic approach. Factor XIII enhances blood clot stability by cross-linking fibrin monomers and integrating alpha 2 -antiplasmin into developing clots. There is no routinely available test parameter that can indicate factor XIII deficiency. In addition, there are currently no standardized reference values. However, especially in diffuse bleeding, factor XIII substitution (15-30 IU/kg) may be indicated to achieve a factor XIII activity above 60-70\% [40].

Administration of recombinant factor VIIa $(90 \mu \mathrm{g} / \mathrm{kg})$ induces a so-called 'thrombin burst'. As the final stage of the therapy algorithm, this approach may potentially reverse life-threatening coagulopathy. However, several conditions, such as plasma $\mathrm{pH} \geq 7.2$, fibrinogen concentration $>150 \mathrm{mg} / \mathrm{dl}$, platelet count $>50,000 / \mu \mathrm{l}$, hematocrit $\geq 25 \%$, ionized calcium concentration $>1 \mathrm{mmol} / \mathrm{l}$ and body temperature $>36{ }^{\circ} \mathrm{C}$, should be met before factor VIIa is administered. Moreover, as highlighted in a recent systematic review, the effectiveness of recombinant factor VIIa in reducing the transfusion rate of allogeneic blood products or perioperative blood loss remains controversial [41].

\section{Conclusion}

In the absence of prospective, randomized trials, adequate data are not yet available regarding the use of POC techniques in critically ill patients in the ICU. Nevertheless, POC coagulation testing is faster and more comprehensive than conventional laboratory tests of blood coagulation, and enables effective and economical 
hemotherapy. None of the POC techniques covers the whole spectrum of hemostasis, thus the combination of aggregometric and viscoelastic methods is recommended. Hemotherapy should be based on an escalating algorithm including POC diagnostics, which is adapted to the specific bleeding risks of the patient's condition (e.g., cardiovascular surgery, organ transplantation, and trauma surgery). In this respect, POC techniques should be an integral part of a patient blood management program [42]; beyond this, POC analysis may also enable diagnosis of sepsis-induced hemostatic alterations, and may even have the potential to predict mortality in septic patients. Finally, prospective studies are urgently needed to analyze whether hemostatic therapy based on POC testing can provide significant benefits with respect to clinical outcomes for our critical care patients.

\section{Competing interests}

KDZ has received speaker's honoraria from CSL Behring. CFW has received speakers' honoraria from CSL Behring, TEM international, Verum Diagnostica $\mathrm{GmbH}$ and Roche AG. The manuscript was written in the absence of any industrial influence or financial support.

\section{List of abbreviations used}

ACT: activated clotting time; ALI: acute lung injury; aPTT: activated partial thromboplastin time; CRP: C-reactive protein; DDAVP: desamino-D-arginine; DIC: disseminated intravascular coagulation; FFP: fresh frozen plasma; HIT: heparin-induced thrombocytopenia; ICU: intensive care unit; IL: interleukin; INR: international normalized ratio; MCF: maximum clot firmness; PC: platelet concentrate; POC: point-of-care; PRBC: packed red blood cells; SAPS: simplified acutre physiology system; SOFA: sequential organ failure assessment.

\section{Published: 19 March 2013}

\section{References}

1. Levi M, Opal SM: Coagulation abnormalities in critically ill patients. Crit Care 2006, 10:222.

2. Glance LG, Dick AW, Mukamel DB, Fleming FJ, Zolla RA, Wissler R, Salloum R, Meredith UW, Osler TM: Association between intraoperative blood transfusion and mortality and morbidity in patients undergoing noncardiac surgery. Anesthesiology 2011, 114:283-292.

3. Murphy GJ, Reeves BC, Rogers CA, Rizvi SI, Culliford L, Angelini GD: Increased mortality, postoperative morbidity, and cost after red blood cell transfusion in patients having cardiac surgery. Circulation 2007, 116:2544-2552

4. Gorlinger K, Bergmann L, Dirkmann D: Coagulation management in patients undergoing mechanical circulatory support. Best Pract Res Clin Anaesthesiol 2012, 26:179-198.

5. Kozek-Langenecker S: Management of massive operative blood loss. Minerva Anestesiol 2007, 73:401-415.

6. Haas T, Spielmann N, Mauch J, Madjdpour C, Speer O, Schmugge M, Weiss M: Comparison of thromboelastometry $(\mathrm{ROTEM}(\mathrm{R}))$ with standard plasmatic coagulation testing in paediatric surgery. Br J Anaesth 2012, 108:36-41.

7. Toulon P, Ozier Y, Ankri A, Fleron MH, Leroux G, Samama CM: Point-of-care versus central laboratory coagulation testing during haemorrhagic surgery. A multicenter study. Thromb Haemost 2009, 101:394-401.

8. Jambor C, von Pape KW, Spannagl M, Dietrich W, Giebl A, Weisser H: Multiple electrode whole blood aggregometry, PFA-100, and in vivo bleeding time for the point-of-care assessment of aspirin-induced platelet dysfunction in the preoperative setting. Anesth Analg 2011, 113:31-39.

9. Hartert $\mathrm{H}$ : Thrombelastography, a method for physical analysis of blood coagulation. Z Gesamte Exp Med 1951, 117:189-203.

10. Avidan MS, Alcock EL, Da Fonseca J, Ponte J, Desai JB, Despotic GJ, Hunt BJ: Comparison of structured use of routine laboratory tests or near-patient assessment with clinical judgement in the management of bleeding after cardiac surgery. Br J Anaesth 2004, 92:178-186.
11. Ak K, Isbir CS, Tetik S, Atalan N, Tekeli A, Aljodi M, Civelek A, Arsan S: Thromboelastography-based transfusion algorithm reduces blood product use after elective CABG: a prospective randomized study. J Card Surg 2009, 24:404-410.

12. Westbrook AJ, Olsen J, Bailey M, Bates J, Scully M, Salamonsen RF: Protocol based on thromboelastograph (TEG) out-performs physician preference using laboratory coagulation tests to guide blood replacement during and after cardiac surgery: a pilot study. Heart Lung Circ 2009, 18:277-288.

13. Weber CF, Gorlinger K, Meininger D, Hermann E, Bingold T, Moritz A, Cohn LH, Zacharowski K: Point-of-care testing: A prospective, randomized clinical trial of efficacy in coagulopathic cardiac surgery patients. Anesthesiology 2012, 117:531-547

14. Nuttall GA, Oliver WC, Santrach PJ, Bryant S, Dearani JA, Schaff HV, Ereth MH: Efficacy of a simple intraoperative transfusion algorithm for nonerythrocyte component utilization after cardiopulmonary bypass. Anesthesiology 2001, 94:773-781.

15. Gorlinger K, Dirkmann D, Hanke AA, Kamler M, Kottenberg E, Thielmann M, Jakob H, Peters J: First-line therapy with coagulation factor concentrates combined with point-of-care coagulation testing is associated with decreased allogeneic blood transfusion in cardiovascular surgery: a retrospective, single-center cohort study. Anesthesiology 2011, 115:1179-1191.

16. Wang SC, Shieh JF, Chang KY, Chu YC, Liu CS, Loong CC, Chan KH, Mandell S, Tsou MY: Thromboelastography-guided transfusion decreases intraoperative blood transfusion during orthotopic liver transplantation: randomized clinical trial. Transplant Proc 2010, 42:2590-2593.

17. Gorlinger K: Coagulation management during liver transplantation. Hamostaseologie 2006, 26:S64-S76.

18. Gorlinger K, Fries D, Dirkmann D, Weber CF, Hanke AA, Schochl H: Reduction of fresh frozen plasma requirements by perioperative point-of-care coagulation management with early calculated goal-directed therapy. Transfus Med Hemother 2012, 39:104-113.

19. Stainsby D, MacLennan S, Thomas D, Isaac J, Hamilton PJ: Guidelines on the management of massive blood loss. Br J Haematol 2006, 135:634-641.

20. Sarani B, Dunkman WJ, Dean L, Sonnad S, Rohrbach JI, Gracias VH: Transfusion of fresh frozen plasma in critically ill surgical patients is associated with an increased risk of infection. Crit Care Med 2008, 36:1114-1118

21. Watson GA, Sperry JL, Rosengart MR, Minei JP, Harbrecht BG, Moore EE, Cuschieri J, Maier RV, Billiar TR, Peitzman AB, Inflammation and Host Response to Injury Investigators: Fresh frozen plasma is independently associated with a higher risk of multiple organ failure and acute respiratory distress syndrome. J Trauma 2009, 67:221-227.

22. Maegele $M$, Lefering $R$, Yucel $N$, et al.: Early coagulopathy in multiple injury: an analysis from the German Trauma Registry on 8724 patients. Injury 2007, 38:298-304

23. Rourke C, Curry N, Khan S, et al:: Fibrinogen levels during trauma hemorrhage, response to replacement therapy, and association with patient outcomes. J Thromb Haemost 2012, 10:1342-1351.

24. Holcomb JB, Minei KM, Scerbo ML, Radwan ZA, Wade CE, Kozar RA, Gill BS, Albarado R, McNutt MK, Khan S, Adams PR, McCarthy JJ, Cotton BA: Admission rapid thrombelastography can replace conventional coagulation tests in the emergency department: experience with 1974 consecutive trauma patients. Ann Surg 2012, 256:476-486.

25. Schochl H, Nienaber U, Hofer G, Voelckel W, Jambor C, Scharbert G, kozekLagenecker S, Solomon C: Goal-directed coagulation management of major trauma patients using thromboelastometry (ROTEM)-guided administration of fibrinogen concentrate and prothrombin complex concentrate. Crit Care 2010, 14:R55.

26. Afshari A, Wikkelso A, Brok J, Moller AM, Wetterslev J: Thrombelastography (TEG) or thromboelastometry (ROTEM) to monitor haemotherapy versus usual care in patients with massive transfusion. Cochrane Database Syst Rev 2011, CD007871.

27. Adamzik M, Eggmann M, Frey UH, Görlinger K, Bröcker-Preuss M, Marggraf G, Saner F, Eggebrecht H, Peters J, Hartmann M: Comparison of thromboelastometry with procalcitonin, interleukin 6, and C-reactive protein as diagnostic tests for severe sepsis in critically ill adults. Crit Care 2010, 14:R178.

28. Adamzik M, Langemeier T, Frey UH, Göttinger K, Saner F, Eggebrecht H, Peters $\mathrm{Jm}$, Hartmann M: Comparison of thrombelastometry with simplified acute physiology score II and sequential organ failure assessment scores for the 
prediction of 30-day survival: a cohort study. Shock2011, 35:339-342.

29. Spalding GJ, Hartrumpf M, Sierig T, Oesberg N, Kirschke CG, Albes JM: Cost reduction of perioperative coagulation management in cardiac surgery: value of "bedside" thrombelastography (ROTEM). Eur J Cardiothorac Surg 2007, 31:1052-1057.

30. Crowther MA, Warkentin TE: Bleeding risk and the management of bleeding complications in patients undergoing anticoagulant therapy: focus on new anticoagulant agents. Blood 2008, 111:4871-4879.

31. Hiippala ST, Myllyla GJ, Vahtera EM: Hemostatic factors and replacement of major blood loss with plasma-poor red cell concentrates. Anesth Analg 1995, 81:360-365.

32. Bolliger D, Gorlinger K, Tanaka KA: Pathophysiology and treatment of coagulopathy in massive hemorrhage and hemodilution. Anesthesiology 2010, 113:1205-1219.

33. Warmuth M, Mad P, Wild C: Systematic review of the efficacy and safety of fibrinogen concentrate substitution in adults. Acta Anaesthesiol Scand 2012, 56:539-548.

34. Dickneite G, Pragst: Prothrombin complex concentrate vs fresh frozen plasma for reversal of dilutional coagulopathy in a porcine trauma model. Br J Anaesth 2009, 102:345-354.

35. Scharbert G, Kalb M, Marschalek C, Kozek-Langenecker SA: The effects of test temperature and storage temperature on platelet aggregation: a whole blood in vitro study. Anesth Analg 2006, 102:1280-1284.

36. Rinder CS, Mathew JP, Rinder HM, Bonan J, Ault KA, Smith BR: Modulation of platelet surface adhesion receptors during cardiopulmonary bypass. Anesthesiology 1991, 75:563-570.
37. Picker SM: In vitro assessment of platelet function. Transfus Apher Sci 2011, 44:305-319.

38. Lethagen S: Desmopressin (DDAVP) and hemostasis. Ann Hemato/ 1994, 69:173-180

39. Cauwenberghs S, van Pampus E, Curvers J, Akkerman JW, Heemskerk JW: Hemostatic and signaling functions of transfused platelets. Transfus Med Rev 2007, 21:287-294.

40. Korte W: F. XIII in perioperative coagulation management. Best Pract Res Clin Anaesthesiol 2010, 24:85-93.

41. Yank V, Tuohy CV, Logan AC, Bravata DM, Staudenmayer K, Eisenhut R, Sundaram V, McMahon D, Olkin I, McDonald KM, Owens DK, Stafford RS: Systematic review: benefits and harms of in-hospital use of recombinant factor VIla for off-label indications. Ann Intern Med 2011, 154:529-540.

42. Shander A, Van Aken H, Colomina MJ, Gombotz H, Hoffman A, Krauspe R, Lasocki S, Richards T, Slappendel R, Spahn DR: Patient blood management in Europe. Br J Anaesth 2012, 109:55-68.

doi:10.1186/cc12527

Cite this article as: Meybohm P, et al:. Point-of-care coagulation management in intensive care medicine. Critical Care 2013, 17:218. 